\title{
Function and Structure in the Diphenylamine-Exposed Kidney
}

\author{
Kenneth D. Gardner, Jr., Sidney Solomon, William W. Fitzgerrel, and Andrew P. Evan \\ with the technical assistance of Priscilla B. Searl and Theresa Chavez
}

From the Departments of Medicine, Physiology, and Anatomy, University of

New Mexico School of Medicine, Albuquerque, New Mexico 87131; and the

Department of Medicine, University of Hawaii School of Medicine,

Honolulu, Hawaii 96822

A B S T R A C T Standard micropuncture and microdissection techniques were used to examine the function and structure of nephrons in rats whose kidneys were made cystic by dietary exposure to diphenylamine. Heterogeneity characterized the lesion, with dilation and frank cyst formation occurring in 5-30\% of nephrons. Elevated intraluminal hydrostatic pressures, occurring in the absence of increased glomerular filtration or decreased net water reabsorption, were recorded in dilated, but not in nondilated nephrons. Structural studies demonstrated communication of dilated nephrons with cysts, concretions of debris within tubular lumens, evidence of extrinsic pressure by cysts on adjacent tubules, and apparent luminal narrowing of some proximal tubules. These observations were used to explain prolonged loop of Henle transit times and occasional failure to detect $\left[{ }^{3} \mathrm{H}\right]$ inulin excretion after microperfusion into dilated tubules. It was concluded that the elevated hydrostatic pressures in the dilated nephrons of diphenylamine-exposed kidneys were the consequence of variably severe and frequently incomplete tubular occlusion. These findings support the hypothesis that cyst formation is a consequence of partial obstruction and elevated intratubular pressure in this model and perhaps in other susceptible mammalian kidneys.

\section{INTRODUCTION}

Numerous explanations have been offered to account for the formation and expansion of cysts in the mammalian kidney $(1,2)$. They include a "blowing up," a "growing out," and a "pulling out" of renal tubular walls. Data

A portion of this work appeared in abstract form ( $J$. Clin. Invest. 1972. 51: 34a).

Received for publication 23 January 1973 and in revised form 3 November 1975. offered in support of these hypotheses have been gathered primarily in careful studies of the morphology of human cystic kidneys. Commonly these organs have been examined in the later stages of their involvement.

From such observations, it has not been possible to deduce the mechanism by which cysts form and expand in the mammalian kidney. Cyst formation does not appear to be a simple case of continuing filtration into blind sacs or occluded tubules inasmuch as cysts can, and in diseases such as polycystic kidney disease usually do, form along nephrons which are not anatomically occluded $(1,3,4)$. The absence of complete occlusion suggests that hydrostatic forces play an insignificant role in cyst formation.

A recent experimental study supports this conclusion. Carone and associates examined structure and function in rat kidneys made cystic by exposure to diphenyl thiazole, a chemical which induces relatively homogeneous dilation of all nephrons (5). In the absence of detectable differences in glomerular function, intratubular pressures, water and solute reabsorption, and obstruction among most tubules in these kidneys, Carone et al. concluded that a defect in tubular basement membrane, which operates in the presence of normal transtubular pressures, is responsible for cyst formation in drug-induced renal cystic disease.

Because of a question whether similar conditions exist in other forms of mammalian renal cystic disease, we examined structure and function in another model, in which cyst formation is induced in rats by chronic dietary exposure to diphenylamine (DPA) ${ }^{\mathbf{1}}(6)$. The lesion produced by this agent differs significantly from that in

\footnotetext{
${ }^{1}$ Abbreviations used in this paper: DPA, diphenylamine GFR, glomerular filtration rate; SNGFR, single nephron glomerular filtration rate; $T F / P_{I n}$, tubular fluid to plasma inulin ratio.
} 
the model of Carone et al. Changes in nephron structure are heterogeneous, not homogeneous. Cyst formation occurs only in a fraction of all nephrons present in a single kidney. In this respect, the model more closely approximates the lesion seen in human polycystic and medullary cystic kidney disease $(3,7)$. For the investigator the DPA model has a practical attribute. Cyst formation depends on the impurity (8) and on the level and duration (9) of exposure to the drug. Consequently, it is possible to prepare and select rats for study not only when cyst formation is advanced, but also during evolution of their cystic renal disease when dilatation of scattered renal tubules is present but frank cyst formation is absent or rare.

Data obtained from the study of DPA-exposed kidneys indicate that increased intraluminal pressure exists in some nephrons in this model and implicates partial or intermittent obstruction as the cause. We are led to a conclusion that increased intraluminal pressure plays an important role in cyst formation in these and perhaps in other susceptible mammalian kidneys.

\section{METHODS}

Male Sprague-Dawley rats weighing $75-100 \mathrm{~g}$ were fed $1 \%$ by weight $\mathrm{DPA}^{2}$ in flavored laboratory chow for 5-20 mo. Experiments were carried out on the kidneys of these and control rats of similar weights taken either from stock or from animals maintained on an identical diet in which methyl cellulose by weight had been substituted for DPA.

Experiments were performed after the rats had been fasted for $18 \mathrm{~h}$ with free access to water. Animals were anesthetized with Inactin (Promonta, Hamburg, W. Germany; $100 \mathrm{mg} / \mathrm{kg}$ body weight intraperitoneally), placed on a heated table to maintain body temperature between $36^{\circ}$ and $38^{\circ} \mathrm{C}$, as monitored rectally by thermacouple (TeleThermometer; Yellow Springs Instrument Co., Yellow Springs, Ohio), and were subjected to tracheostomy and jugular venous catheterization. An infusion of Ringer's solution was begun at $4-6 \mathrm{ml} / \mathrm{h}$ and allowed to run for $90 \mathrm{~min}$ to permit equilibration. Nephron micropuncture was carried out by the general technique described by Barenberg et al. (10). Micropipettes were prepared as described by Vurek et al. (11) to 3-18 $\mu \mathrm{m}$ OD and were inserted under visual guidance with micromanipulators (E. Leitz, Inc., Rockleigh, N. J.) and a dissecting microscope with fiber optic illumination (American Optical Corp., Southbridge, Mass.). When functional studies were completed, kidneys from some animals were taken at random and submitted for anatomical study.

Under these general conditions the following series of observations were made:

(a) Tubular diameters. In each experiment, the in vivo diameter of individual cortical nephrons was assessed visually and recorded as "dilated" or "nondilated" (normal diameter). A representative number of nephrons was chosen at random and their luminal diameters were measured directly by using a filar micrometer eyepiece (Bausch \&

${ }^{2}$ Obtained from Eastman Kodak Co., Rochester, N. Y., and incorporated into pellet form by General Biochemicals, Inc., Chagrin Falls, Ohio.
Lomb, Inc., Rochester, N. Y.). Diameters were expressed in microns.

(b) Intratubular hydrostatic pressures. Two methods were utilized to measure intraluminal pressures. The first was that of Gottschalk and Mylle, utilizing direct aqueous manometry (12). 6-12 manometric readings were made in each nephron by a second observer, who recorded on call from the experimentor at those instants when a $1 \%$ lissamine green-Ringer's solution neither entered nor left the tip of the micropipette. No data were recorded unless the tip was clearly visible in the tubular lumen. Readings were corrected for the capillarity of the micropipette and averaged to arrive at a mean value for each nephron studied.

Pressures also were measured by utilizing the pressuresensitive servo-nulling device described by Brenner et al. (13) to monitor pressure before and during the collection of tubular fluid in studies of single nephron glomerular filtration rate (SNGFR; vide infra).

(c) SNGFR. SNGFR's were determined as described by Barenberg et al. (10). In these series of experiments $\left[{ }^{3} \mathrm{H}\right]$ inulin (New England Nuclear, Boston, Mass.) was added to the infusion to yield a concentration of $25 \mu \mathrm{Ci} / \mathrm{ml}$. Samples of ureteral urines were collected (bilaterally in most experiments) at 15 -min intervals. Tail blood was sampled at the beginning, middle, and end of each experiment. Radioactivity in tubular fluid, urine, and serum was measured with a liquid scintillation counter (Packard Instrument Co., Inc., Downers Grove, Ill.). Timed free-flow samples collected from individual nephrons were transferred to scintillation fluid for counting. SNGFR's were calculated by dividing total counts in each sample by the product of the plasma inulin concentration and the duration of the collection period. Total kidney glomerular filtration rates (GFR) were calculated similarly using timed urine collections.

(d) Tubular fluid to plasma inulin ratios $\left(T F / P_{I n}\right)$. Free flow samples of tubular fluid were collected as for SNGFR. Samples were extruded under oil onto a glass depression slide. Known volumes of tubular fluid ( 5 or more nanoliters) were recovered with precalibrated pipettes and were transferred to scintillation vials for counting. Samples of tail vein blood were obtained at $15-\mathrm{min}$ intervals and counted as above.

(e) Recovery of $\left[{ }^{3} \mathrm{H}\right]$ inulin after single nephron microinjection. After insertion of the micropipette, consecutive 10-min urine samples were collected from bilateral ureteral catheters. By using a calibrated microperfusion pump (Wolfgang Hampel, Berlin, W. Germany), known amounts of Ringer's solution containing $1 \%$ lissamine green and $\left[{ }^{3} \mathrm{H}\right]-$ inulin in a concentration sufficient to yield 85 counts $/ \mathrm{nl} \cdot \mathrm{min}$ (approximately three-times background) were perfused into individual nephrons over a $2-\mathrm{min}$ period at $25 / \mathrm{nl} \cdot \mathrm{min}$. This pump was shown capable of delivering a constant amount of perfusate against a hydrostatic resistance of $60 \mathrm{~cm}$ water applied at the delivery end of a micropipette mounted in a specially designed chamber. Consecutive 10 -min urine collections were continued after each perfusion until radioactivity in urine from both kidneys returned to the preperfusion baseline.

$\left[{ }^{3} \mathrm{H}\right]$ Inulin recovery was expressed as a percent of counts recovered in ureteral urine from the left (perfused) and right (nonperfused) kidneys relative to the total amount of $\left[{ }^{3} \mathrm{H}\right]$ inulin injected. In four experiments, single microperfusions were performed in individual animals and urine collections were continued for 70-90 min.

Oil blockade. In a separate series of five control rats perfusions were performed in seven unobstructed tubules and in six tubules in which a droplet of oil $6-8$ diameters in 

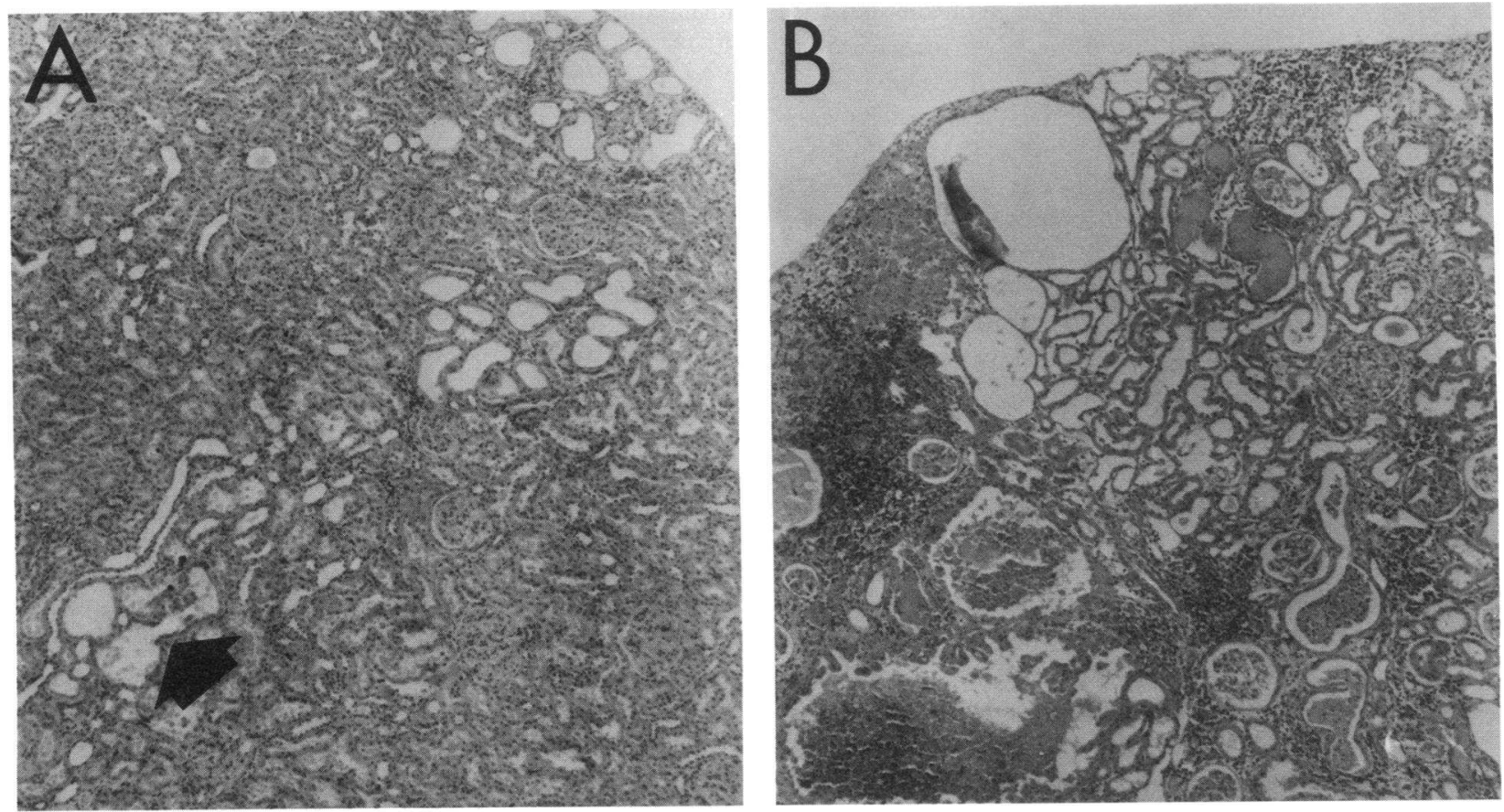

FIGURE 1 The DPA-exposed rat kidney. The typical renal lesion after $8 \pm 3$ mo of dietary exposure to $1 \%$ DPA is shown to the left (A). Note focal tubular dilation extending through the cortex to the kidney surface (upper right). The arrow highlights the abrupt change in tubular diameter. The cortical changes which typify the renal lesion after $15 \pm 3$ mo of $1 \%$ DPA exposure are shown in 1B. Frank cyst formation is evident. Scattered lumens are filled with particulate or colloidal debris. $(16 \times)$

length preceded entrance of the dye-isotope solution into the nephron. Urine was collected from both ureters for the ensuing $45 \mathrm{~min}$ and counts excreted by the left kidney were computed by the formula $100 \times[(L+R)-2 R] / L+R$ in which $L=$ counts excreted by the left kidney; $R=$ counts excreted by the right kidney; and $2 R$ was taken as an index of recirculated $\left[{ }^{3} \mathrm{H}\right]$ inulin. After instillation of oil and label, pressures were measured by manometry in the same tubules.

(f) Loop of Henle transit times. The interval between the initial entrance of perfusate into a surface nephron and its appearance in the distal convolution of the same nephron (loop transit time) was measured by stopwatch in 18 dilated and 28 nondilated nephrons in 13 rats fed DPA for 5-11 mo and in 22 nephrons of 6 normal rats. Results were recorded in seconds.

( $g$ ) Statistical analyses. Data from each of these series of experiments were analyzed statistically, initially by Student's $t$ test. If significance was not achieved and when a marked variation in the magnitude of readings was present, the Mann-Whitney $U$ test was used to examine rank order significance (14).

(h) Microscopic and microdissection studies. When functional studies were completed, kidneys from some animals were selected at random and submitted for anatomical study. An aqueous solution of latex particles was injected from a micropipette inserted at the point of initial micropuncture. These nephrons subsequently were dissected by the method of Oliver and Macdowell (15) or were sought in unstained sections cut serially from paraffin-imbedded tissue. Light and scanning electron microscopy were performed in routine fashion on tissue from additional kidneys.

\section{RESULTS}

At the end of the feeding period, body weights did not differ between DPA-fed and control rats (range, 304 $500 \mathrm{~g}$ for each group). Two groups of animals were established arbitrarily for convenience in examining the data. The first contained those rats fed DPA for 5-11 mo in which alterations in renal morphology were less marked. Dilated tubules in these animals were considered "precystic." The second contained those rats fed DPA for 12-20 mo. Dilated tubules and cysts were present in their kidneys.

The weights of kidneys relative to body weight did not differ significantly, averaging ( $\pm \mathrm{SE}$ ) $0.90 \pm 0.03 \%$ in normals, $0.88 \pm 0.02 \%$ in the $5-11$-mo DPA-fed group, and $0.84 \pm 0.02 \%$ in the $12-20$-mo DPA-fed rats.

Heterogeneity characterized both kidney structure and function. Nephrons in varying stages of dilation and cystic deformity were present in all DPA-exposed kidneys. The microscopic appearances of typical kidneys exposed to DPA for 5-11 mo and 12-20 mo, respectively, are shown in Fig. 1.

(a) Tubular diameters. The number of dilated tubules which were visible on the DPA-exposed kidneys varied from one or two in some kidneys to innumerable in 
others. Longer exposure to DPA was associated with a larger number of visibly dilated tubules. Luminal diameters ranged between 34.2 and $110.8 \mu \mathrm{m}$ in 22 dilated nephrons and between 20.6 and $45.1 \mu \mathrm{m}$ in 10 undilated nephrons in DPA-exposed kidneys. In six nephrons of two control rats, tubular diameter ranged between 25.9 and $34.2 \mu \mathrm{m}$. The differences in diameters between dilated and nondilated nephrons were significant $(P<$ 0.001 ) by both methods of statistical analyses.

(b) Intraluminal hydrostatic pressures. Visibly dilated surface proximal convoluted tubules had intraluminal pressures that were significantly higher than the pressures recorded in nondilated nephrons in the same kidneys (Table I). This difference was documented by each of the two techniques employed to measure pressure. Pressures measured by the servo-nulling transducer technique tended to be higher, but the technique was applied only in the 12-20-mo group. Significant differences existed among control, early, and late DPA-exposed kidneys in the rank order of their magnitude ( $U$ test) not in their arithmetical means ( $t$ test). There was one exception: pressures measured by direct manometry averaged a significantly higher value in dilated nephrons of rats fed DPA for 5-11 mo. Among normal rats, the difference between mean pressures measured by manometry and by transducer was not statistically significant.

(c) SNGFR. Whole kidney (left) GFR as a function of total body weight did not differ significantly among the groups of kidneys studied (means in $\mathrm{ml} / \mathrm{min}$. $\mathrm{kg}$ body weight $\pm \mathrm{SE}$ ) : $3.88 \pm 0.42$ in normal rats, $3.30 \pm$ 0.41 in 8 rats fed DPA for $5-11 \mathrm{mo}$, and $3.10 \pm 0.35$ in 13 rats fed DPA for $12-20$ mo. Statistically significant differences were not found in SNGFR's (Table II). In six rats fed DPA for 12-20 mo, intraluminal pressures were monitored during the collection of samples of

TABLE I

Intraluminal Pressures in Renal Tubules of DPA-Fed and Normal (Control) Rats

\begin{tabular}{lcccc}
\hline & $\begin{array}{c}\text { No. } \\
\text { of } \\
\text { rats }\end{array}$ & $\begin{array}{c}\text { No. } \\
\text { of } \\
\text { tubules }\end{array}$ & Range & Mean \pm SE \\
\hline & & & $c m H_{2} \mathrm{O}$ & \\
Water manometry & & & & \\
DPA dilated* & 7 & 30 & $5.3-44.8$ & $26.2 \pm 1.6 \neq$ \\
DPA nondilated* & 7 & 24 & $4.2-31.5$ & $19.3 \pm 0.8$ \\
Control & 2 & 11 & $3.9-24.8$ & $20.6 \pm 1.8$ \\
& & & & \\
Servo-nulling transducer & & & & \\
DPA dilated & 6 & $10 \|$ & $28.2-167.9$ & $52.1 \pm 13.59$ \\
DPA nondilated & 5 & $13 \|$ & $0.1-55.7$ & $29.7 \pm 5.0$ \\
Control & 11 & 49 & $4.6-72.3$ & $21.4 \pm 1.9$ \\
\hline
\end{tabular}

* In rats fed DPA for 5-11 mo.

‡ Significantly different from other values in group; $P<0.05, t$ test.

\& In rats fed DPA for 12-20 mo.

\| Same nephrons studied for SNGFR (see Table II).

I Significantly different from other values in group; $P<0.05, U$ test.
TABLE II

SNGFR'S in Renal Tubules of DPA-Fed and Normal (Control) Rats

\begin{tabular}{|c|c|c|c|c|}
\hline & $\begin{array}{l}\text { No. } \\
\text { of } \\
\text { rats }\end{array}$ & $\begin{array}{c}\text { No. } \\
\text { of } \\
\text { tubules }\end{array}$ & Range & Mean \pm SE* \\
\hline & & & \multicolumn{2}{|c|}{$n l / \min$} \\
\hline \multicolumn{5}{|c|}{ DPA-fed 5-11 mo } \\
\hline Dilated & 9 & 26 & $7.1-121.9$ & $62.1 \pm 6.7$ \\
\hline Nondilated & 9 & 30 & $6.4-131.6$ & $56.6 \pm 2.7$ \\
\hline \multicolumn{5}{|c|}{ DPA-fed $12-20 \mathrm{mo}$} \\
\hline Dilated & 5 & $13 \ddagger$ & $22.9-91.4$ & $50.6 \pm 6.8$ \\
\hline Nondilated & 6 & $10 \ddagger$ & $13.3-99.6$ & $48.4 \pm 7.8$ \\
\hline Control & 25 & 92 & $0.4-133.0$ & $47.2 \pm 2.9$ \\
\hline
\end{tabular}

* Differences not significant.

¥ Same tubules in which pressures were monitored (Table I).

SNGFR. SNGFR's were found not to be related to initial pressure, mean collection pressure, or to variation in collection pressures in either normal or DPA-exposed kidneys (Fig. 2).

(d) $T F / P_{\text {In }}$. TF $/ \mathrm{P}_{\text {In's }}$ ' were measured in rats fed DPA and in normal animals. No significant difference was found between the two groups of rats nor between the two populations of nephrons in kidneys exposed to DPA for 5-11 mo. In the 12-20-mo group, however, $\mathrm{TF} / \mathrm{P}_{\text {In's }}$ were significantly increased (Table III).

(e) Recovery of $\left[{ }^{3} \mathrm{H}\right]$ inulin after single nephron microinjection. The data from a typical experiment in this series are plotted in Fig. 3. Placement of the micropipette

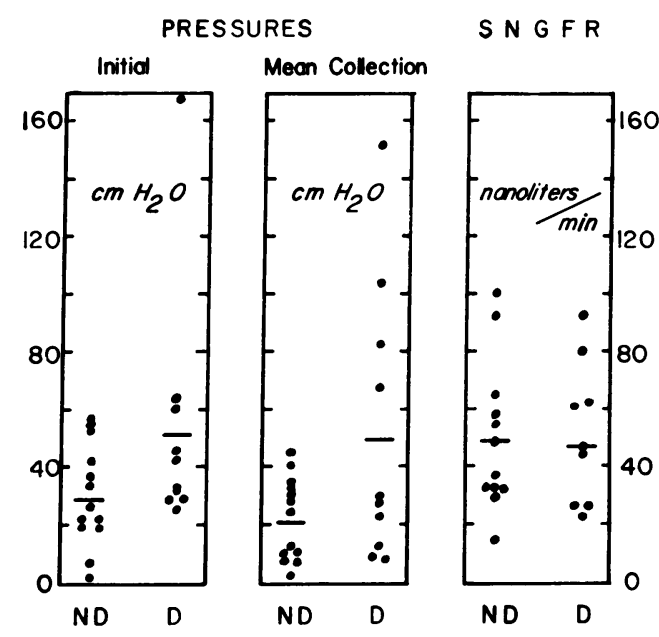

FIGURE 2 Initial and mean collection pressures (average, beginning, and ending pressures, plus pressure reading at four intervals of $20,40,60,80 \%$ ) during collection for SNGFR in 13 nondilated (ND) and 10 dilated (D) tubules of six 12-20-mo DPA-fed rats. Cross bars represent mean values. Differences in mean collection pressures were shown by analyses of variance to be due to original differences in pressure.

The Diphenylamine-Exposed Kidney 
TABLE III

$T F / P_{I n}$ in Renal Tubules of DPA-Fed and Normal (Control) Rats

\begin{tabular}{lcccc}
\hline & $\begin{array}{c}\text { No. } \\
\text { of } \\
\text { rats }\end{array}$ & $\begin{array}{c}\text { No. } \\
\text { of } \\
\text { tubules }\end{array}$ & Range & Mean \pm SE \\
\hline DPA-fed rats 5-11 mo & & & & \\
$\quad$ Dilated & 4 & 11 & $2.87-8.26$ & $3.89 \pm 0.75$ \\
$\quad$ Nondilated & 4 & 25 & $1.41-5.24$ & $3.14 \pm 0.25$ \\
DPA-fed rats 12-20 mo & & & & \\
$\quad$ Dilated & 4 & 20 & $2.89-11.40$ & $6.06 \pm 0.59 *$ \\
$\quad$ Nondilated & 4 & 18 & $3.14-8.40$ & $4.47 \pm 0.57 \ddagger$ \\
$\quad$ Control rats & 3 & 23 & $1.49-6.72$ & $2.91 \pm 0.22$ \\
\hline
\end{tabular}

* Significantly different from all but 12-20-mo nondilated nephrons; $P<0.05$.

† Significantly different from control rats only ; $P<0.05$.

containing isotope-dye into a nephron characteristically was followed by a short burst of radioactivity in the urines in both kidneys. This was allowed to subside before the perfusion pump was turned on. After injection of normal and nondilated tubules, radioactivity usually appeared promptly in left ureteral urine and subsided quickly. In contrast, placement of the micropipette in a dilated tubule rarely was followed by a burst of radioactivity and perfusion of such a nephron resulted in the recovery of less isotope.

The results of all $\left[{ }^{3} \mathrm{H}\right]$ inulin recovery studies are summarized in Table IV. In none of the three groups was a significant amount of $\left[{ }^{3} \mathrm{H}\right]$ inulin recovered in urine from the right kidney. Detectable radioactivity emerged from the right side in 10 of 20 perfusions of nephrons in DPA-exposed kidneys. No detectable increase in radioactivity was found in right ureteral urine after perfusion of nephrons in normal control kidneys.

The range of values for inulin recoveries was extremely wide after perfusion of dilated nephrons (0.4 $93.3 \%)$. It contrasted sharply with the ranges recorded for nondilated (72.0-121.2\%) and normal (74.4-130.5\%) nephrons. In four experiments in which collections were extended for 70-90 min after microperfusion of one dilated nephron in a single kidney, inulin recoveries averaged $1.4,23.1,2.9$, and $30.8 \%$, respectively, in urine from the perfused kidney. No detectable isotope appeared in the blood nor in right ureteral urine (with one exception) of any of these animals.

Oil blockade. Dye-isotope excretion from unblocked tubules was prompt; green dye appeared promptly in the urine of all seven experiments. Excreted counts average $83.4 \pm 2.9 \%$ total and ranged from 74.6 to $94.6 \%$ of radioactivity recovered in the urine over $45 \mathrm{~min}$. Among these tubules pressures average $24.7 \pm 2.1$ and ranged from 15.2 to $33.4 \mathrm{~cm} \mathrm{H}_{2} \mathrm{O}$. In contrast among oil-blockaded tubules green urine never appeared subsequent to micro- perfusion, excreted counts averaged $-6.5 \pm 1.5 \%$ and ranged from -1.2 to $-12.1 \%$ (i.e., $2 R$ exceeded the sum of $L+R$ ), and pressures averaged $40.7 \pm 1.3$ and ranged between 35.8 and $44.0 \mathrm{~cm} \mathrm{H}_{2} \mathrm{O}$. The differences in counts excreted $(P<0.001)$ and pressures $(P<0.001)$ were significant.

(f) Loop of Henle transit times. Transit time data are summarized in Table $V$. The mean period of time required for a lissamine green solution to pass from injection site to the earliest visible loop of distal tubule was prolonged 3-4 times in dilated nephrons. The end point often was difficult, sometimes impossible, to detect accurately. The difference in transit times between nondilated and normal nephrons was far smaller but also was significant statistically.

Transit times and $\left[{ }^{3} \mathrm{H}\right]$ inulin recoveries were recorded in the same nephrons in 21 instances during this study (in nine dilated, eight nondilated, and four normal nephrons). Longer transit times were associated with reduced inulin recovery to a significant degree (correlation coefficient $=0.648 ; P<0.01$ ). Tubular diameters were measured along with transit times on 14 occasions (in seven dilated and seven nondilated nephrons of five rats fed DPA for 5-11 mo), and a direct and significant

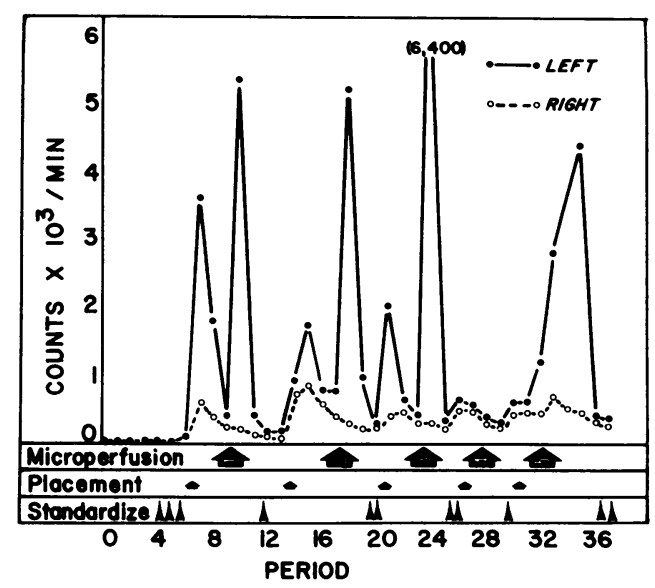

Figure 3 Data from an $\left[{ }^{3} \mathrm{H}\right]$ inulin recovery experiment in an 11-mo DPA-exposed precystic kidney. Urinary radioactivity is indicated on the ordinate and consecutively numbered $10-\mathrm{min}$ collection periods on the abscissa. Illustrated is the sequence of steps-standardization of the pipette, placement in tubules, and perfusion-which was followed in these experiments. $\left[{ }^{3} \mathrm{H}\right]$ Inulin recovery was derived from the counts recovered in ureteral urine during the two successive 10-min collection periods after perfusion and expressed as a percentage of total counts injected. In this animal, perfusions $1,2,3$, and 5 were into nondilated tubules while 4 was into a dilated tubule. $\left(\left[{ }^{3} \mathrm{H}\right]\right.$ Inulin recovery was $6 \%$ of total counts injected after perfusion 4.) In this experiment, data from perfusions 3 and 5 were rejected because of an error in perfusion speed and admixture of two left ureteral urine specimens, respectively. 

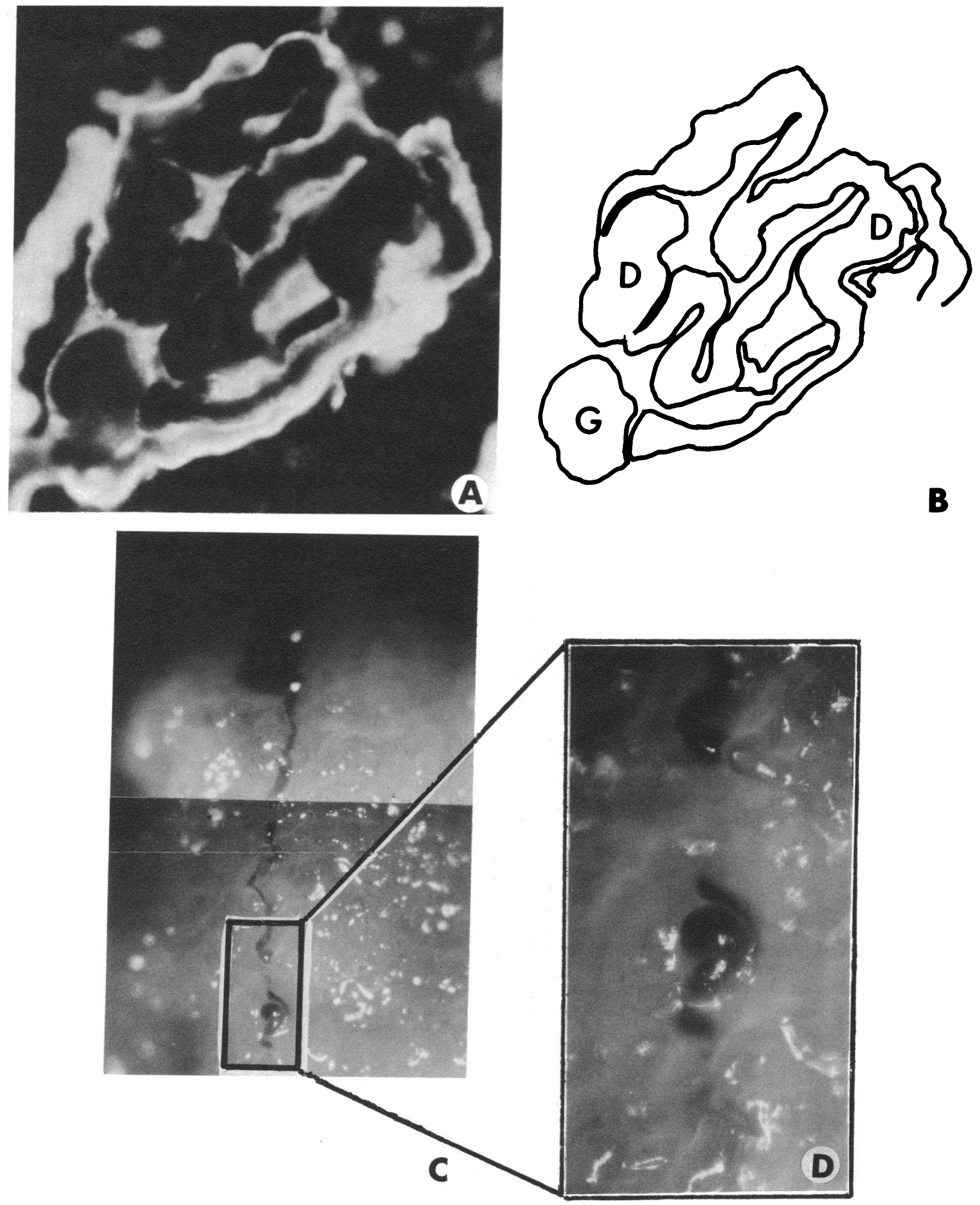

FIGURE 4 (A) A representative latex-filled nephron dissected from a precystic kidney (indirect illumination, approximately $125 \times$ ). (B) Tracing of nephron shown in A, indicating glomerulus (G) and loci of tubular dilations (D). (C and D) Low-powered photomicrograph of teased surface of a 14-mo DPA-exposed kidney showing appearance of single latex-filled nephron. Capsular surface is toward the top. Note mass of dilated cortical proximal convoluted tubule coils at surface; pars recta, with approximately normal diameter; and latex-filled cyst deep within renal substance. 

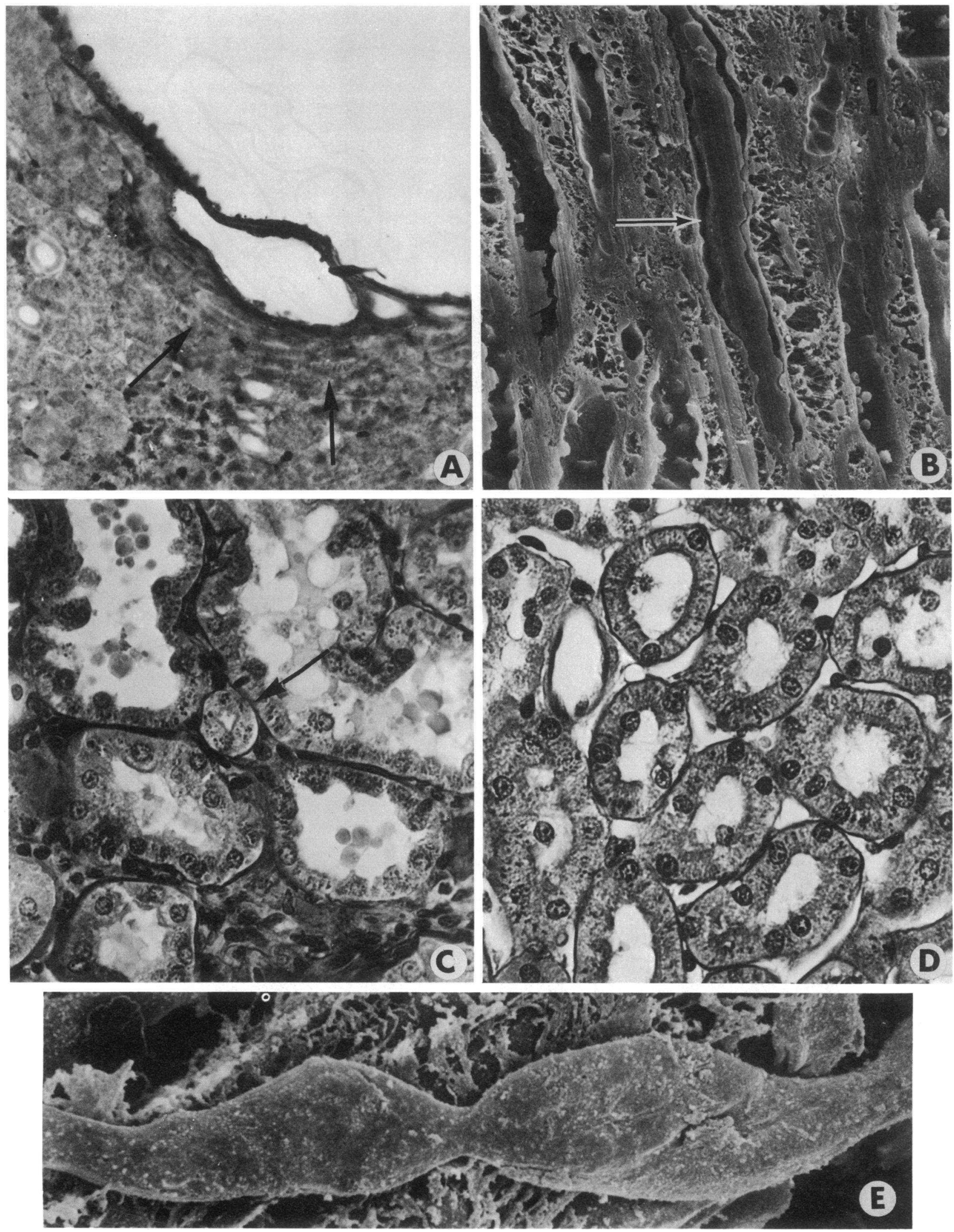
TABLE IV

$\left[{ }^{3} \mathrm{H}\right]$ Inulin Recovery from Left and Right Ureteral Urines after Perfusion of Single Nephrons in Left Kidney of Normal and 11 DPA-Fed Rats

\begin{tabular}{|c|c|c|c|c|}
\hline & \multirow{2}{*}{$\begin{array}{c}\text { No. of } \\
\text { nephrons }\end{array}$} & \multirow{2}{*}{$\begin{array}{c}\text { Range of } \\
\text { observation } \\
\text { periods }\end{array}$} & \multicolumn{2}{|c|}{$\begin{array}{c}\text { Mean } \pm \mathrm{SE} \%\left[^{[3} \mathrm{H}\right] \text { inulin } \\
\text { recovered }\end{array}$} \\
\hline & & & Left & Right \\
\hline & & $\min$ & & \\
\hline \multicolumn{5}{|l|}{ DPA-fed rats } \\
\hline Dilated & 11 & $40-90$ & $29.9 \pm 8.2$ & $6.7 \pm 3.4^{*}$ \\
\hline Nondilated & 9 & $30-70$ & $93.4 \pm 5.7 \ddagger$ & $1.4 \pm 5.7^{*}$ \\
\hline Control rats & 6 & $20-70$ & $100.8 \pm 9.8 \ddagger$ & $0.4 \pm 0.04$ \\
\hline
\end{tabular}

* Not significantly different from zero.

$\ddagger P$ value of difference from dilated nephrons: $<0.001$.

correlation was found (correlation coefficient $=0.745$; $P<0.02)$.

( $g$ ) Microscopic and microdissection studies. In general terms, observations confirmed the changes in renal morphology which have been described by others in the DPA-exposed kidney $(6,8,9)$. Five observations were made which were considered to have specific relevance to the functional studies described above: Along proximal and collecting tubules dilation was shown to be segmental, not diffuse (Figs. 4A and 5E). Dilated tubules at the surface occasionally communicated with cysts deep in the renal substance (Fig. 4B). Adjacent to intrarenal cysts, tubules often appeared compressed (Fig. 5A). Dilated collecting tubules often contained debris which appeared to be partially occluding their lumens (Fig. $5 B$ ). And DPA-exposed kidneys possessed proximal convoluted tubules with segments of apparent narrowing (Fig. 5C). In none of the nephrons or the microscopic sections studied was complete occlusion of a tubular lumen identified.

\section{DISCUSSION}

DPA produces a renal lesion in rats which characteristically is heterogeneous in its severity and focal in its distribution (6). In the rats whose kidneys are reported here $5-30 \%$ of surface nephrons appeared visibly dilated in vivo, an appraisal that was confirmed by measurements of tubular diameter. Usually, dilated nephrons were more prevalent at the poles than in the central por-
TABLE V

Visual Proximal to Distal Tubule Transit Times in 13 DPA-Exposed and 6 Normal Kidneys

\begin{tabular}{lcc}
\hline & $\begin{array}{c}\text { No. of } \\
\text { nephrons }\end{array}$ & $\begin{array}{c}\text { Mean } \pm \mathrm{SE} \\
\text { transit time }\end{array}$ \\
\hline DPA-exposed rats & & $s$ \\
Dilated & 18 & $98.1 \pm 8.0$ \\
Nondilated & 28 & $28.9 \pm 1.4^{*}$ \\
Control rats & 22 & $23.5 \pm 1.5 \ddagger$ \\
\hline
\end{tabular}

* $P$ value of difference from dilated nephrons: $<0.001$. $\ddagger P$ value of difference from nondilated nephrons: $<0.05$.

tion of the kidney. No ready explanation is available for this observation.

Our results indicate that, under the conditions described, intraluminal pressures are elevated in the dilated surface nephrons of the DPA-exposed kidney. Two possible explanations for this elevation are ruled out by the measurements of SNGFR's and $\mathrm{TF} / \mathrm{P}_{\mathrm{In}}$ ratios. Were elevated pressures the result of sustained "super-filtration," one might have expected higher SNGFR's. They were not found; SNGFR's, including those measured under conditions of monitored pressure, were not significantly different among dilated versus nondilated nephrons in either early or late disease. Were elevated pressures the result of a chronic reduction in net water reabsorption, one would have expected lower $\mathrm{TF} / \mathrm{P}_{\mathrm{In}}$ ratios in dilated nephrons. In actuality, equal to higher $\mathrm{TF} / \mathrm{P}_{\mathrm{In}}$ ratios were found.

Persisting filtration into totally occluded nephrons also could account for elevated intraluminal pressures. The evidence against this alternative is both morphological and functional: No completely occluded nephrons were found by microdissection or microscopic examination, an experience identical to that of earlier workers $(2-4$, $9)$. Lissamine green and $\left[{ }^{3} \mathrm{H}\right]$ inulin, placed into dilated segments of proximal nephrons, usually traversed the loop to appear only in ipsilateral urine. Complete occlusion, therefore, clearly does not exist in all dilated nephrons of the DPA-exposed kidney. Consequently it cannot be considered a prerequisite to elevated intraluminal pressure and nephron dilation.

Figure 5 (A) Medium-power microscopy of area adjacent to large corticomedullary cyst (lumen upper right), showing compression of tubules (arrows) adjacent to a dilated nephron $(1,100 \times)$. (B) Scanning electron microscopic view of a cross-section of renal medulla from DPA-exposed kidney showing dilated collecting tubule with a lumen partially occluded by debris (arrow). (C and D) Renal cortex from DPA-fed (C) and normal (D) rats. Note "minitubule" in the DPA-exposed kidney. Its brush border identifies it as a proximal tubule. Tubules of similar diameter were not observed in kidneys from control rats. Latex particles in such tubules indicated they communicated with dilated, micropunctured surface nephrons. (E) Scanning electron microscopic view of isolated collecting tubule from 14-mo DPA-exposed rat showing two foci of dilation adjacent to segments of normal diameter $(400 \times)$. 
A fourth possibility to account for elevated pressure is partial or intermittent occlusion. Microscopic sections and dissected nephrons were examined for sites of narrowing and partial obstruction, a more subjective and difficult task than the identification of dilated tubules. Fig. 5 exemplifies the nature of the morphological evidence found: narrowed tubules, intraluminal casts and debris, and compression of nephrons by adjacent cysts. Cysts, by compressing adjacent nephrons in vivo (Fig. $5 \mathrm{~A}$ ), could cause their partial or intermittent occlusion while in vitro subsequent microdissection would reveal what appeared to be perfectly patent tubules.

These considerations led us to conclude that elevated pressures in the dilated surface nephrons of the DPAexposed kidney most likely are the consequence of partial and/or intermittent downstream occlusion. This conclusion was reinforced by the observation that pressures were elevated and the excretion of the dye-isotope label was reduced or eliminated in normal tubules that were intentionally blocked by droplets of oil.

Some of our results suggest that partial or intermittent occlusion may be relatively widespread and progressive in the DPA-exposed kidney. Although two different techniques were used to measure pressures (water manometry and servo-nulling transducer), the highest pressures were encountered among dilated tubules in the most advanced stages of the lesion. $\mathrm{TF} / \mathrm{P}_{\mathrm{In}}$ ratios were relatively higher among the more severely dilated nephrons in longer standing disease. Transit times were prolonged significantly even among nondilated nephrons in the earlier lesion. And the number of visibly dilated surface nephrons increased with the duration of DPAexposure.

Three aspects of this study require further comment. The first deals with label "disappearance," a phenomenon observed in a minority of the microperfusion experiments. The second concerns the finding that SNGFR's tended to remain unchanged in the face of increased intraluminal pressure. The third concerns the quantitative significance of our data.

The appearance of labels in urine was not invariable. Lissamine green injection and $\left[{ }^{3} \mathrm{H}\right]$ inulin microperfusion occasionally was followed by apparent "disappearance" of the markers. Failure of the microperfusion pump was ruled out when in vitro observations demonstrated its ability to deliver accurate volumes against as much as 60 $\mathrm{cm}$ water pressure applied to the tip of its micropipette. Entrapment of the labels in medullary tissue by countercurrent forces seemed implausible for several reasons: The efficiency of the countercurrent mechanism is impaired in DPA-induced nephropathy (9). Dye staining of medullary tissue was not observed after sectioning of injected kidneys. And $\left[{ }^{3} \mathrm{H}\right]$ inulin recirculation, an event considered likely to accompany inulin escape from a mi- croperfused nephron, did not occur regularly or even frequently, as evidenced by the absence of significant increases in the levels of blood and contralateral urine radioactivity in all but a rare instance of label disappearance.

Coupled with these considerations was the fact that morphological studies provided evidence in support of a more plausible, although speculative, explanation for label "disappearance." Label could be trapped or delayed in nephrons compressed by adjacent cysts or in eddy currents arising along dilated segments of tubules. Dilated surface nephrons were shown (Figs. 4 and 5) to communicate with both compressed nephrons and frank cysts deep within the renal substance. Some cysts measured in excess of $2,500 \mu \mathrm{m}$ in diameter. Assuming spherical configuration in vivo, they would have contained over 2,000 $\mathrm{nl}$ of fluid, more than enough to dilute the 4,250 counts perfused in $50 \mathrm{nl}$ to a level of radioactivity only 2-3 counts above background. A change this small would have gone undetected.

The observation that SNGFR's tended to remain unchanged despite higher pressures in dilated nephrons is compatible with current theory and implies an intrinsic change in glomerular function. Deen et al. (16) have indicated that glomerular filtration is determined by transglomerular capillary hydrostatic and osmotic gradients and by the functional permeability of the glomerular membrane. Were intratubular pressure to rise, thereby narrowing the hydrostatic gradient across the capillary wall, SNGFR could remain unchanged only were the transglomerular osmotic gradient to narrow or the functional permeability of the glomerular membrane to increase. We have no direct information as to which of these two alternatives occurs in the DPA-exposed kidney but have observed obliteration of glomerular foot-processes in the lesion (A. P. Evan, unpublished observation). This morphological change suggests increased glomerular permeability to protein. A glomerular protein leak, by narrowing the transglomerular osmotic gradient, could offset the narrowed pressure gradient across the glomerulus and allow SNGFR's to be maintained.

The heterogeneity of nephron morphology and the wide ranges over which data from the DPA-exposed kidneys often were spread have important implications in the analyses and interpretation of our results. Save for observations made under technically unsatisfactory circumstances, no reading was discarded because it lay more than two or three standard deviations away from the mean of its group. Ignorance of the extent to which heterogeneity of function as well as structure might exist in these nephrons led us to a decision to include all observations. This meant that occasionally a widely discrepant value was included, e.g., the precollection pressure of $167.9 \mathrm{~cm} \mathrm{H}_{2} \mathrm{O}$ in a dilated, DPA-exposed nephron 
(Fig. 2). Statistical significance was sought in differences of rank order (the $U$ test) as well as of group means (the $t$ test) to accommodate this variability. Under such circumstances and especially in light of the frequently skewed distribution of data, arithmetical means were given less quantitative significance than traditionally is given them in studies of the homogeneous, normal kidney.

Although data are scant, there is reason to believe that increased intraluminal pressure plays an important role in cystic kidney disease. The case rests on the facts that elevated pressures are found in cystic kidneys and that elevated intratubular pressures lead to cystic deformity.

In addition to the DPA-exposed kidney, elevated intraluminal pressures also occur in the diphenyl thiazole model, albeit in "less than 5\%" of nephrons (5). Variably increased pressures have been recorded in vivo among cysts in human polycystic kidney disease (17). Thus, to date elevated intraluminal pressures have been documented in each variety of cystic kidney disease which has been examined.

The changes produced in nephron morphology by ureteral obstruction, a procedure which elevates intraluminal pressure $(12,13)$ evidently by retarding drainage from nephrons with persisting glomerular filtration, have been studied by Strong, Fetterman, and Shimamura and their respective co-workers $(18-20)$. Tubular dilation is not diffuse but segmental, may involve not all but only a portion of nephrons in the obstructed kidney, and is characterized as "cystic" in appearance. It occurs as an acquired lesion in a presumed previously normal kidney, a condition also met by the chemically induced models. But in contrast to these models, nephron dilation and cyst formation in the obstructed kidney occurs in the absence of exposure to any exogenous cystogenic substance.

Currently, increased compliance of the tubular wall is an alternative hypothesis to account for cyst formation (5). The conclusion has been established by default. It is based on a lack of evidence for obstruction in a majority of nephrons in the diphenyl thiazole-exposed model (5) and demonstrations that metabolic factors can at least accelerate nephron dilation and cyst formation (9). However, no direct studies of compliance in nephrons from cystic kidneys are available. There is no basis for an assumption that the cellular (degenerative) and peritubular (thickened basement membrane) changes which characterize early DPA-induced nephropathy $(2,8,9)$ increase rather than decrease or leave unchanged the compliance of the tubular wall. And the studies of ureteral obstruction already cited demonstrate that cyst formation can occur without any postulated initial change in mural compliance, itself a postulated consequence of the action of a cystogenic chemical.
On the bases of published experience with ureteral obstruction and our demonstrations of increased intraluminal pressure and delayed excretion of dye and isotope from dilated nephrons, we hypothesize that the primary lesion in the DPA-exposed kidney is an obstructive one, caused by tubular narrowing, intraluminal debris, and in the later stages by the compression of otherwise normal tubules from adjacent dilating nephrons. We attribute cyst formation in this model to obstruction and a subsequent elevation of intraluminal pressures that arise as the consequence of sustained glomerular filtration and unaltered net water reabsorption in the involved nephrons.

It is tempting to extrapolate these findings and conclusions to human renal cystic disease. While the DPA model and human cystic renal disease share several points in common (heterogeneity in structural deformity $[3,4]$, apparent squeezing of adjacent otherwise patent and normal nephrons [3], variably elevated pressures in tubules or cysts of the same kidney [17], and apparent patency of dilated and cystic nephrons [3]), there is no reason to assume that the pathogenesis of cyst formation is identical in the model and in man. However, it seems likely that data from the study of human material will continue to be relatively scarce, particularly since pretransplant nephrectomy has become unfashionable. Careful study of animal models, at the moment, appears to be the major approach available in order to achieve some understanding of the pathogenesis of cystic renal disease in a susceptible mammalian kidney.

\section{ACKNOWLEDGMENTS}

The authors are grateful to Elizabeth Skipper, Ph.D., for her assistance with statistical analyses, and to Patricia Carlton for preparation of histological sections.

This work was supported by Public Health Service grants HL 13137, HL 16775, and AM HL 17641.

\section{REFERENCES}

1. Osathanondh, V., and E. L. Potter. 1964. Pathogenesis of polycystic kidneys. Historical survey. Arch. Pathol. 77: 459-465.

2. Darmady, E. M., J. Offer, and M. A. Woodhouse. 1970. Toxic metabolic defect in polycystic disease of the kidney. Evidence from microscope studies. Lancet. 1: 547-550.

3. Lambert, P. P. 1947. Polycystic disease of the kidney. A review. Arch. Pathol. 44: 34-58.

4. Osathanondh, V., and E. L. Potter. 1964. Pathogenesis of polycystic kidneys. Survey of results of microdissection. Arch. Pathol. 77 : 510-512.

5. Carone, F. A., R. G. Rowland, S. G. Perlman, and C. E. Ganote. 1974. The pathogenesis of drug-induced renal cystic disease. Kidney Int. 5: 411-421.

6. Thomas, J. O., A. J. Cox, Jr., and F. DeEds. 1957. Kidney cysts produced by diphenylamine. Stanford Med. Bull. 15: 90-93.

The Diphenylamine-Exposed Kidney 
7. Goldman, S. H., S. R. Walker, T. C. Merigan, Jr., K. D. Gardner, Jr., and J. M. C. Bull. 1966. Hereditary occurrence of cystic disease of the renal medulla. N. Engl. J. Med. 274: 984-992.

8. Crocker, J. F. S., D. M. Brown, R. F. Borch, and R. L. Vernier. 1972. Renal cystic disease induced in newborn rats by diphenylamine derivatives. $\mathrm{Am} . \mathrm{J}$. Pathol. 66: 343-350.

9. Safouh, M., J. F. S. Crocker, and R. L. Vernier. 1970 Experimental cystic disease of the kidney. Sequential, functional and morphologic studies. Lab. Invest. 23: 392-400.

10. Barenberg, R. L., S. Solomon, S. Papper, and R. Anderson. 1968. Clearance and micropuncture study of renal function in mercuric chloride treated rats. J. Lab. Clin. Med. 72: 473-484.

11. Vurek, G. G., C. M. Bennett, R. L. Jamison, and J. L. Troy. 1967. An air-driven micropipette sharptener. $J$. Appl. Physiol. 22 : 191-192.

12. Gottschalk, C. W., and M. Mylle. 1956. Micropuncture study of pressures in proximal tubules and peritubular capillaries of the rat kidney and their relation to ureteral and renal venous pressures. Am. J. Physiol. 185: 430439.

13. Brenner, B. M., J. L. Troy, and T. M. Daugharty. 1972. Pressures in cortical structures of the rat kidney. $\mathrm{Am}$. J. Physiol. 222 : 246-251.
14. Mann, H. B., and D. R. Whitney. 1947. On a test of whether one of two random variables is stochastically larger than the other. Ann. Math. Stat. 18: 50-60.

15. Oliver, J., and M. MacDowell. 1961. The structural and functional aspects of the handling of glucose by the nephrons and the kidney and their correlation by means of structural-functional equivalents. J. Clin. Invest. 40: 1093-1112.

16. Deen, W. M., C. R. Robertson, and B. M. Brenner. 1972. A model of glomerular ultrafiltration in the rat. Am. J. Physiol. 223 : 1178-1183.

17. Bjerle, P., B. Lindqvist, and G. Michaelson. 1971. Pressure measurements in renal cysts. Scand. J. Clin. Lab. Invest. $27: 135-138$.

18. Strong, K. C. 1940. Plastic studies in abnormal renal architecture. V. The parenchymal alterations in experimental hydronephrosis. Arch. Pathol. 29: 77-119.

19. Fetterman, G. H., M. M. Ravitch, and F. E. Sherman. 1974. Cystic changes in fetal kidneys following ureteral ligation: studies by microdissection. Kidney Int. 5: 111-121.

20. Shimamura, T., J. M. Kissane, and F. Györkey. 1966. Experimental hydronephrosis. Nephron dissection and electron microscopy of the kidney following obstruction of the ureter and in recovery from obstruction. Lab. Invest. 15 : 629-640. 